\title{
Efecto de la concentración de Ga sobre las propiedades electrónicas del $\mathrm{CuIn}_{1-X} \mathrm{Ga}_{X} \mathrm{Se}_{2}$
}

\author{
Ga Concentration Effect on the $\mathrm{CuIn}_{1-x} \mathrm{Ga}_{x} \mathrm{Se}_{2}$ Electronic Properties
}

\author{
D. A. Rasero Causil ${ }^{\mathrm{a}, *}$ \\ A. A. Portacio Lamadrid ${ }^{\text {b }}$ \\ J. A. Rodríguez ${ }^{\mathrm{c}}$
}

Recepción: 04-sep-2015

Aceptación: 20-ene-2016

\begin{abstract}
Resumen
Se reportan cálculos de propiedades electrónicas del compuesto $\mathrm{CuIn}_{1-x} \mathrm{Ga}_{x} \mathrm{Se}_{2}(x=0,0 ; 0,2 ; 0,4 ; 0,6$; $0,8 ; 1,0)$, usando el método Tight-Binding (TB) y Virtual Crystal Approximation (VCA). Se considera el caso ideal y con las distorsiones tetragonal $(\eta)$ y aniónica $(\mu)$. En ambos casos, el $\mathrm{CuIn}_{1-x} \mathrm{Ga}_{x} \mathrm{Se}_{2}$ es un semiconductor directo en $\Gamma$, para todas las concentraciones. Se encontró que el Crystal Field Splitting (CFS) en el punto $\Gamma$ depende principalmente de la distorsión tetragonal. El CFS es positivo para $x<0,32$ y negativo para $x>0,32$. Este comportamiento se debe a que cuando aumenta $x$, la celda unitaria se contrae, acercando el pseudoátomo (In,Ga) al átomo de Se.
\end{abstract}

Palabras clave: $\mathrm{CuIn}_{1-x} \mathrm{Ga}_{x} \mathrm{Se}_{2}$, Tight-Binding, aproximación de cristal virtual, Crystal Field Splitting.

\begin{abstract}
This paper reports some calculations of the electronic properties of $\mathrm{CuIn}_{1-x} \mathrm{Ga}_{x} \mathrm{Se}_{2}(x=0.0,0.2,0.4,0.6$, $0.8,1.0$ ) compound, by using the Tight-Binding (TB) method and Virtual Crystal Approximation (VCA). It is considered the ideal case and with the tetragonal $(\eta)$ and anionic $(\mu)$ distortions. In both cases, the $\mathrm{CuIn}_{1-x} \mathrm{Ga}_{x} \mathrm{Se}_{2}$ is a direct semiconductor at $\Gamma$, for all concentrations.It was found that the Crystal Field Splitting (CFS) at the $\Gamma$ point depends mainly on the tetragonal distortion. The CFS is positive for $x<0,32$ and negative for $x>0.32$. This behavior is due that when $\mathrm{x}$ is increasing, the unit cell shrinks, approaching the pseudo-atom $(\mathrm{In}, \mathrm{Ga})$ to the $\mathrm{Se}$ atom.
\end{abstract}

Key words: $\mathrm{CuIn}_{1-x} \mathrm{Ga}_{x} \mathrm{Se}_{2}$, Tight-Binding, Virtual Crystal Approximation, Crystal Field Splitting.

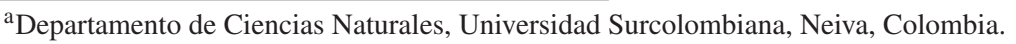

*Autor de correspondencia: diegorasero@gmail.com

${ }^{\mathrm{b}}$ Departamento de Matemáticas y Física, Universidad de los Llanos, Villavicencio, Colombia.

${ }^{\mathrm{c}}$ Departamento de Física, Universidad Nacional de Colombia, Bogotá, Colombia.
} 


\section{Introducción}

Entre las diferentes fuentes de energía, la solar es una buena elección frente a los combustibles fósiles, ya que no contamina el ambiente y se puede utilizar durante gran parte del año. Para aprovechar esta o cualquier otra fuente de energía se necesita del generador adecuado. Los generadores fotovoltáicos son una alternativa de producción de energía eléctrica; con estos se puede complementar la energía generada, por ejemplo, por hidroeléctricas, que están siendo limitadas por la influencia del clima, de las lluvias, y últimamente afectadas por fenómenos de cambio climático; también se puede complementar la energía de termoeléctricas, que consumen combustible fósil, agotando las reservas y, además, contaminan el medioambiente. Con el compuesto ternario $\mathrm{CuInSe}_{2}$ (CIS) en estructura calcopirita (ver figura 1) se han construido celdas solares fotovoltáicas de bajo costo y eficiencias cercanas al 16\% [1]. Aleando los semiconduntores $\mathrm{CuInSe}_{2}$ (de gap directo $1,04 \mathrm{eV}$ ) y $\mathrm{CuGaSe}_{2}$ (de gap directo $1.70 \mathrm{eV}$ ) en estructura policristalina se pueden construir celdas con eficiencias cercanas al 19\% [2-7]; esta aleación, conocida como $\mathrm{CuIn}_{1-x} \mathrm{Ga}_{x} \mathrm{Se}_{2}$ (CIGS), conforma la capa absorbente de la celda.

Los resultados experimentales [8] muestran que el gap de energías prohibidas $E_{g}$ de esta aleación varía cuadráticamente con la concentración $x$ de Ga. El parámetro de curvatura (bowing parameter) experimental es $b_{\exp }=0,107 \mathrm{eV}$. En un reporte previo [9] hemos calculado los parámetros Tight-Binding (TB) que reproducen esta dependencia cuadrática y la estructura electrónica para la concentración $x=0,6$.

En este trabajo calculamos la estructura electrónica y la variación del Crystal Field Splitting (CFS) principal del CuIn $\mathrm{In}_{1-x} \mathrm{Ga}_{x} \mathrm{Se}_{2}$ para las concentraciones de $\mathrm{Ga}, x=0,0 ; 0,2 ; 0,4 ; 0,6 ; 0,8$ y 1,$0 ;$ usando el método TB y Virtual Crystal Approximation (VCA).

\section{Aspectos teóricos}

\subsection{Estructura cristalina}

La celda unitaria del CuInSe $2, \mathrm{CuIn}_{1-x} \mathrm{Ga}_{x} \mathrm{Se}_{2}$ y $\mathrm{CuGaSe}_{2}$ es tetragonal centrada en el cuerpo, con ocho átomos, como se muestra en la figura 1. En el caso ideal cada catión (anión) está en el centro de un tetraedro determinado por sus vecinos cercanos y $c=2 a$. En un caso más real se pueden considerar dos distorsiones: la distorsión aniónica $\mu$, que implica que los aniones no están exactamente en el centro de dichos tetraedros, y la distorsión tetragonal $\eta=c / 2 a$, que impílica $c \neq 2 a$. Según esto, en el caso ideal $\mu=0,25$ у $\eta=1$.

Para $\mathrm{CuInSe}_{2} \mu=0,224$ y $\eta=1,004$, para $\mathrm{CuGaSe}_{2} \mu=0,248$ у $\eta=0,982$.

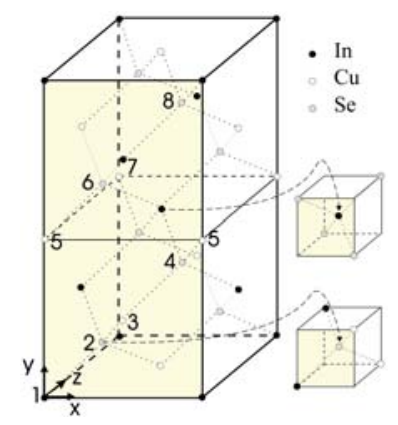

Figura 1. Estructura cristalina tipo calcopirita del $\mathrm{CuInSe}_{2}$.

\subsection{Método Tight-Binding}

El hamiltoniano se construye bajo el modelo TB de Slater and Koster [10], con la modificación de Blom et al. [11] y de Rodríguez [12, 13], en el cual se usa la base de funciones deBloch:

$$
\phi_{\vartheta}^{\alpha}(\vec{k}, \vec{r})=\frac{1}{\sqrt{N}} \sum_{\vec{\tau}} e^{\overrightarrow{i k} \bullet\left(\vec{\tau}+\vec{d}_{\alpha}\right)} \psi_{\vartheta}^{\alpha}\left(\vec{r}-\vec{\tau}-\vec{d}_{\alpha}\right)
$$

En esta expresión, $\vartheta$ identifica los orbitales atómicos; $\alpha$, los átomos de la celda, y $\vec{d}_{\alpha}$ representa la posición del átomo $\vartheta ; \psi_{\vartheta}^{\alpha}$ son orbitales atómicos localizados y $\vec{k}$ es el vector de onda. Se toman orbitales $s$ y $p$ para In, $\mathrm{S}$ y Se, y orbitales $s, p$ y $d$ para $\mathrm{Cu}$. No se considera la interacción spin-órbita. Las amplitudes de transición del electrón de un órbital a otro están dadas por

$$
\begin{aligned}
\int \phi_{\vartheta}^{\alpha *}(\vec{k}, \vec{r}) H \phi_{\vartheta}^{\alpha^{\prime}}, & \left(\overrightarrow{k^{\prime}}, \overrightarrow{r^{\prime}}\right) d^{3} r=\delta_{\vec{k}, \vec{k}}, \times \\
& \sum_{\vec{d}_{\alpha \alpha^{\prime}}} e^{i \vec{k} \bullet \vec{d}_{\alpha \alpha^{\prime}}}\left\langle\vartheta \mid \vartheta^{\prime}\right\rangle_{\vec{d}_{\alpha \alpha^{\prime}}}
\end{aligned}
$$

donde

$$
\left\langle\vartheta \mid \vartheta^{\prime}\right\rangle_{\vec{d}_{\alpha \alpha^{\prime}}}=\int \psi_{\vartheta}^{\alpha *}(\vec{r}) H \psi_{\vartheta^{\prime}}^{\alpha^{\prime}}\left(\vec{r}-\vec{d}_{\alpha \alpha^{\prime}}\right) d^{3} r
$$

La suma que aparece en (2) involucra únicamente interacciones hasta primeros vecinos. La matriz 
hamiltoniana que resulta tiene dimensión $42 \times 42$ y se diagonaliza numéricamente, a lo largo de algunas direcciones de alta simetría de la primera zona de Brillouin (PZB).

\subsection{Virtual Crystal Approximation}

En esta aproximación se considera la aleación como una estructura periódica ordenada. Se adopta la descripción de Hill [14], en la cual el desorden de corto alcance no juega un papel importante en la variación del gap $E_{g}$ con la concentración $x$. Bajo esta aproximación se considera que en $\mathrm{CuIn}_{1-x} \mathrm{Ga}_{x} \mathrm{Se}_{2}$ existe un pseudo-átomo ( $\mathrm{In}, \mathrm{Ga})$. Para $x=0,0$ se tiene $\mathrm{CuInSe}_{2}$, y para $x=1,0, \mathrm{CuGaSe}_{2}$. Se propone, según Olgin [15], que el parámetro $s$ del (In,Ga) varíe de la siguiente manera:

$$
E_{s}^{(I n, G a)}(x)=(1-x) E_{s}^{I n}+x E_{s}^{G a}+x^{2} \Omega_{\mathrm{VCA}}
$$

Donde $E_{s}^{I n}$ y $E_{s}^{G a}$ son los parámetros on-sites del In y $\mathrm{Ga}$ en el CuInSe $\mathrm{y}_{2}$ y en el $\mathrm{CuGaSe}_{2}$, respectivamente; $\Omega_{\mathrm{VCA}}$ es el parámetro VCA de curvatura, introducido para reproducir el comportamiento cuadrático de la curva experimental $E_{g}(x)$, propuesto de la siguiente forma:

$$
\Omega_{\mathrm{VCA}}=k \frac{\left|E_{s}^{I n}-E_{s}^{G a}\right|^{\lambda}}{\left|V_{S S}^{(I n-S e)}-V_{s s}^{(G a-S e)}\right|}
$$

$V_{S S}^{(I n-S e)}$ y $V_{s S}^{(G a-S e)}$ son los parámetros de interacción entre orbitales $s$, a primeros vecinos, de In y Se en el CuInSe 2 , y de Ga y Se en el $\mathrm{CuGaSe}_{2}$, respectivamente. Se propone que los parámetros $p$ del $(\mathrm{In}, \mathrm{Ga})$ varíen linealmente con $x$, esto es:

$$
E_{p}^{(I n, G a)}(x)=(1-x) E_{p}^{I n}+x E_{p}^{G a}
$$

Para determinar $\Omega_{\mathrm{VCA}}$ se realiza un proceso de minimización de la función:

$$
\sigma(k, \lambda)=\sum_{\mathrm{i}}\left\{E_{g}^{\exp }\left(x_{i}\right)-E_{g}^{T B}\left(x_{i}\right)\right\}^{2}
$$

Donde $E_{g}^{\exp }\left(x_{i}\right)$ y $E_{g}^{T B}\left(x_{i}\right)$ son los valores del gap, experimental y teórico (usando la aproximación TB y VCA) para la concentración $x_{i}$, respectivamente.

\section{Resultados y Discusión}

\subsection{Gap de energías prohibidas}

En la figura 2 se muestra la variación de $E_{g}$ como una función de la concentración de Ga. Los rectángulos indican el resultado experimental [2]; con círculosy triángulos se indican los resultados teóricos, calculados en un trabajo previo con el método Tight-Binding [9], para el caso ideal y con distorsiones, respectivamente.

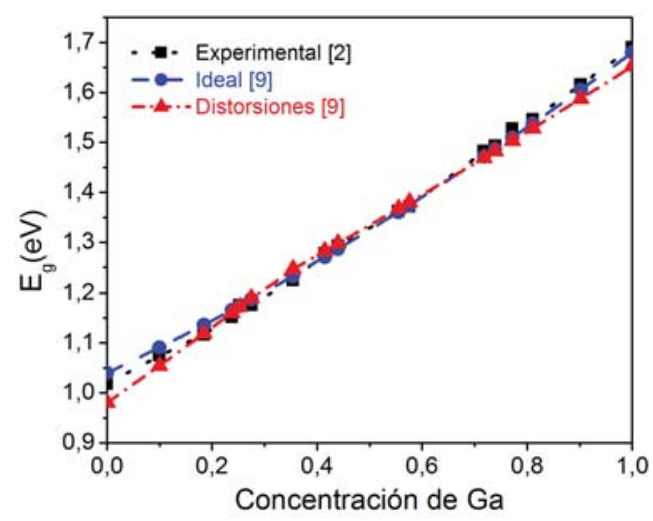

Figura 2. Variación del gap de energías prohibidas en función de la concentración de Ga.

$E_{g}$ varía de manera cuadrática con $x$ [9]. Los datos experimentales indican que

$$
E_{g, \exp }(x)=1,015+0,569 x+0,107 x^{2}
$$

Los parámetros VCA de curvatura para el caso ideal y con distorsiones obtenidos según nuestros cálculos son $\Omega_{V C A \text {,ideal }}=-0,29204 \mathrm{eV}$ y $\Omega_{V} C A$, dist $=-0,13219 \mathrm{eV}$, con los que se obtiene

$$
\begin{array}{r}
E_{g, \text { Ideal }}(x)=1,039+0,500 x+0,141 x^{2} \\
E_{g, \text { Distor }}(x)=0,986+0,7415 x-0,084 x^{2}
\end{array}
$$

Como se observa en la figura 2, nuestros resultados concuerdan bastante bien con los datos experimentales.

\subsection{Estructura de bandas de energía}

Los cálculos de estructura electrónica se muestran en la figura 3, en la que se comparan las bandas ideales (línea continua) y con distorsiones (línea discontinua) para las concentraciones de $\mathrm{Ga}, x=0,0$; 0,$2 ; 0,4 ; 0,6 ; 0,8$ y 1,0 .

El cero de la escala de energías se ha colocado en el máximo de la banda de valencia (BV). Las primeras 26 regiones energéticas corresponden a la $\mathrm{BV}$, y las 16 restantes, a la banda de conducción (BC). 

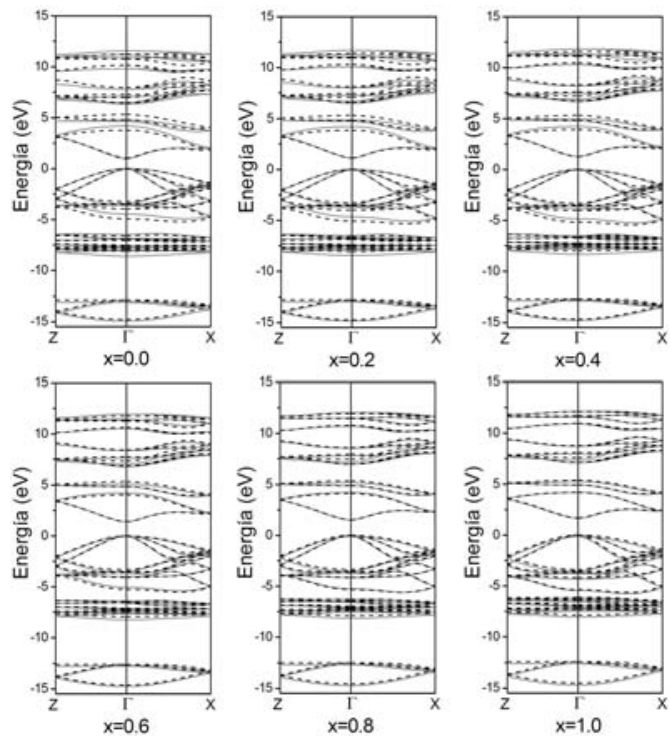

Figura 3. Bandas de energía a lo largo de algunos caminos de alta simetría de la PZB. En línea continua se presentan las bandas en el caso ideal, y en línea discontinua, las bandas con distorsiones.

Nuestros resultados indican que tanto en el caso ideal como con distorsiones, el $\mathrm{CuIn}_{1-x} \mathrm{Ga}_{x} \mathrm{Se}_{2}$ es un semiconductor directo en $\Gamma$, para todas las concentraciones. Este resultado concuerda con el de Rodríguez, para $x=0,0$ y $x=1,0,[12,13]$

En ambos casos, el borde inferior de la BC sube, aumentando el valor del gap. La forma de la parte superior de la BV y de la inferior de la BC, cerca del punto $\Gamma$, es parabólica, y para cada concentración no varía de un caso a otro, indicando que la masa efectiva de los portadores de carga no cambia.

\subsection{Crystal field Spliting principal en $\Gamma$}

En los semiconductores tipo zinc-blenda (ZB) el borde superior de la BV es un triplete (debido a los orbitales $p$-anión, que predominan en esta zona). Las calcopiritas presentan dos cationes en nuestro caso, el $\mathrm{Cu}$ y el pseudo-átomo (In, Ga); por tal razón, el triplete se desdobla en un singlete y un doblete. La diferencia de energía entre estos, $\Delta_{\mathrm{CFS}}=E_{\text {doblete }}-E_{\text {singlete }}$, se llama Crystal Field Splitting principal (CFS). El valor del CFS se debe físicamente a tres causas $[16,17]$ : la existencia de dos cationes, la distorsión tetragonal $\eta$ y la distorsión aniónica $\mu$.

En la figura 4 se muestra la variación del CFS como una función de $x$.

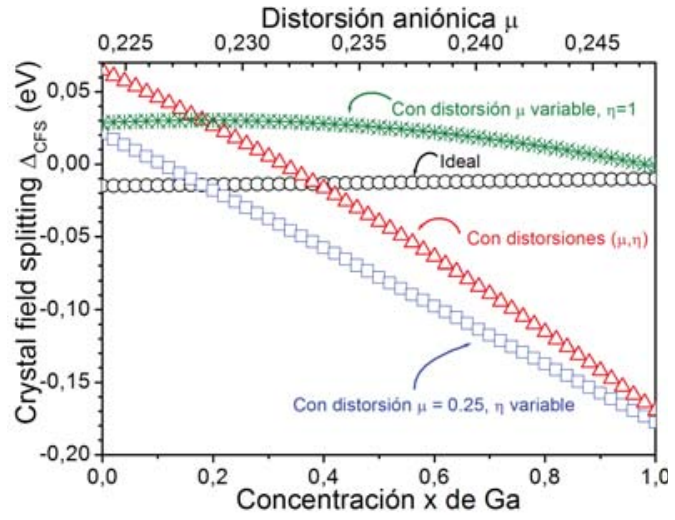

Figura 4. Variación del CFS en función de la concentración de Ga.

Para el caso ideal (círculos), el CFS se mantiene aproximadamente constante y aparece por la existencia de los cationes $\mathrm{Cu}$ e (In, Ga). Además, siempre es negativo, indicando que el singlete está por encima del doblete.

La pequeña variación del CFS se debe al cambio que experimenta la constante de red y los parámetros TB del (In,Ga) a medida que la concentración cambia. Cuando se consideran las distorsiones (triángulos), el CFS varía cuadráticamente con $x$, de acuerdo con el ajuste (en eV):

$$
\Delta_{C F S}=0,065-0,184 x-0,051 x^{2}
$$

Esta variación se debe principalmente a los cambios que sufren las distorsiones $\eta$ y $\mu$ cuando $x$ varía. Para la concentración $x=0,32(\mu=0,232, \eta=0,997)$, $\Delta_{C F S}=0$, indicando que el borde superior de la BV es un triplete. Para $x<0,32$, el CFS es positivo, mientras que para $x>0,32$ el CFS es negativo. El cambio de posición del doblete y el singlete se muestran en la figura 5 .

En la figura 4 también se muestra como cambia el CFS cuando $\mu$ (en asterísticos) y $\eta$ (en rectángulos) varían de manera independiente. Esto indica que la distorsión tetragonal es la principal responsable de la variación del CFS.

En términos de la posición de los átomos en la celda unitaria (ver figura 1), el efecto de la distorsión $\eta$ consiste en disminuir la longitud de enla ceSe(In,Ga). Así, a medida que la concentración de Ga aumenta, la interacción entre estos átomos también aumenta, dando origen al cambio de signo del CFS. 

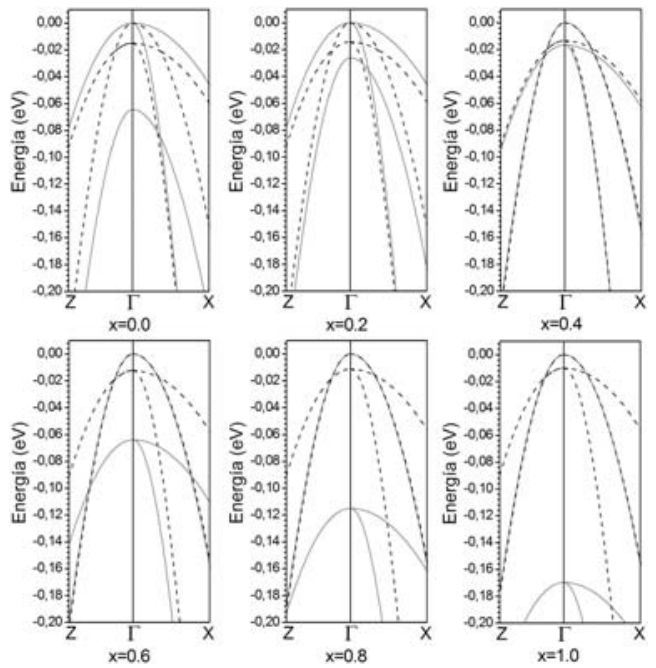

Figura 5. Cambio de posición del doblete y singlete en la parte superior de la BV para las concentraciones de Ga estudiadas. En línea continua se muestra el caso ideal y con línea discontinua el caso con distorsiones.

\section{Conclusiones}

El estudio del compuesto $\mathrm{CuIn}_{1-x} \mathrm{Ga}_{x} \mathrm{Se}_{2}$ para las concentraciones $x=0,0-1,0$, usando TB y VCA, muestra que este material es semiconductor directo en $\Gamma$. El CFS varía cuadráticamente con $x$. Para el caso ideal, el CFS siempre es negativo, y para el caso con distorsiones cambia de signo, lo cual se debe a la interacción de los átomos de Se con el pseudo-átomo $(\mathrm{In}, \mathrm{Ga})$.

\section{Agradecimientos}

A la Universidad Nacional de Colombia, por su apoyo financiero durante el desarrollo de este trabajo.

\section{Referencias}

[1] J. Hedstrom, H. Ohlsen, M. Bodegard, A. Kylner, L. Stolt, D. Hariskos, M. Ruckh, and W. Schock, " $\mathrm{ZnO} / \mathrm{CdS} / \mathrm{Cu}(\mathrm{In}, \mathrm{Ga}) \mathrm{Se}_{2}$ thin film solar cells with improved performance", in Proceedings of the 23rd IEEE Photovoltaic Specialists Conference, pp. 364-371, May 1993.

[2] M. Contreras, B. Egaas, K. Ramanathan, J. Hiltner, A. Swartzlander, F. Hasoon, and R. Noufi, "Progress toward 20\% efficiency in $\mathrm{Cu}(\mathrm{In}, \mathrm{Ga}) \mathrm{Se}_{2}$ polycrystalline thin-film solar cells", Prog. Photovolt: Res. Appl., vol. 7, pp. 311-316, August 1999.
[3] M. Contreras, A. M. Gabor, A. L. Tenant, S. Asher, J. Tuttle, and R. Noufi, "Accelerated publication $16.4 \%$ total-area conversion efficiency thin-film polycrystalline $\mathrm{MgF}_{2} / \mathrm{ZnO} / \mathrm{CdS} / \mathrm{Cu}(\mathrm{In}, \mathrm{Ga}) \mathrm{Se}_{2} / \mathrm{Mo}$ solar cell", Prog. Photovolt: Res. Appl., vol. 2, pp. 287-292, October 1994.

[4] J. R. Tuttle, M. A. Contreras, A. M. Gabor, K. R. Ramanathan, A. L.Tennant, D. S. Albin, J. Keane, and R. Noufi, "Perspective on High-efficiency $\mathrm{Cu}(\mathrm{In}, \mathrm{Ga}) \mathrm{Se}_{2}$-based Thin-film Solar Cells Fabricated by Simple, Scalable Processes", Prog. Photovolt: Res. Appl., vol. 3, 383-391, November 1995.

[5] J. R. Tuttle, J. S. Ward, A. Duda, T. A. Berens, M. A. Contreras, K. R. Ramanathan, A. L. Tennant, J. Keane, E. D. Cole, K. Emery, and R. Nouri, "The Performance of $\mathrm{Cu}(\mathrm{In}, \mathrm{Ga}) \mathrm{Se}_{2}-$ Based Solar Cells in Conventional and Concentrator Applications", Mater. Res. Soc. Symp., vol. 426, 143-151, 1996.

[6] J. R. Tuttle, M. Contreras, A. Tennant, D. Albin, and R. Noufi, "High efficiency thin-film $\mathrm{Cu}$ (In, Ga) $\mathrm{Se}_{2}$-based photovoltaic devices: progress towards a universal approach to absorber fabrication", in Proceedings of the 23rd IEEE Photovoltaic Specialists Conference, pp. 415-421, May 1993.

[7] K. R. Ramanathan, M. A. Contreras, C. L. Perkins, S. Asher, F. S. Hasoon, J. Keane, D. Young, M. Romero, W. Metzger, R. Noufi, J. S. Ward, and A. Duda, "Properties of 19.2\% efficiency $\mathrm{ZnO} / \mathrm{CdS} / \mathrm{CuInGaSe} 2$ thin-film solar cells", Prog. Photovolt: Res. Appl., vol.11, pp. 225-230, June 2003.

[8] R. W. Birkmire, J. R. Sites, "Polycrystalline compound thin film devices: Laboratory cells to modules", in: IEEE (Ed.), 28th IEEE PVSC (Photovoltaic Specialist Conference), IEEE, Anchorage, AK, USA, 2-11, 2000.

[9] T. Suárez, D. Rasero, R. Jiménez, and J. Arbey Rodríguez, " $\mathrm{Cu}(\mathrm{InGa}) \mathrm{Se}_{2}$ : Un estudio de la estructura electrónica usando Tight-Binding, Aproximación de Cristal Virtual y Método de Montecarlo", Rev. Col. Fis., vol. 41, pp. 268270, abril 2009.

[10] J. C. Slater, and G. F. Koster, "Simplified LCAO Method for the Periodic Potential Pro- 
blem", Phys. Rev., vol. 94, pp. 1498-1524, June 1954.

[11] F. A. P. Blom, "Determination of the Fermi Surface in CdSnAs using a Tight-Binding Model for Chalcopyrites", Phys. Rev. B, vol. 32, pp. 2334-2336, August 1985.

[12] J. A. Rodríguez, "Estructura de Bandas de las Calcopiritas basadas en Cobre", Tesis doctoral, Departamento de Física, Universidad Nacional de Colombia, Ciudad Universitaria, Bogotá, D. C., 1999.

[13] J. A. Rodríguez, L. Quiroga, A. Camacho, and R. Baquero, "Electronic Band Structure of $\mathrm{CuInSe}_{2}$ : Bulk and (112) surface", Phys. Rev. $B$, vol. 59, pp. 1555-1558, January 1999.

[14] R. Hill, "Energy-gap Variations in Semiconductor Alloys", J. Phys. C: Solid State Phys., vol. 7, pp. 521-526, February 1974.

[15] R. D. Olguín, "Estructura Electrónica de Compuestos Semiconductores Nuevos II-VI: Superficies y Heteroestructuras", Tesis doctoral, Departamento de Física, Centro de Investigaciones y de Estudios Avanzados del IPN (CINVESTAV), México, D. F., México, 1996.

[16] J. Jaffe, A. Zunger, "Electronic Structure of the Ternary Chalcopyrite Semiconductors $\mathrm{CuAlS}_{2}$, $\mathrm{CuGaS}_{2}, \mathrm{CuInS}_{2}, \mathrm{CuAlSe}_{2}, \mathrm{CuGaSe}_{2}$, and $\mathrm{CuInSe}_{2}$ ", Phys. Rev. B, vol. 28, pp. 58225847, November 1983.

[17] J. Jaffe, and A. Zunger, "Theory of the Bandgap Anomaly in $\mathrm{ABC}_{2}$ Chalcopyrite Semiconductors", Phys. Rev. B, vol. 29, pp. 1882-1906, February 1984. 